\section{A phlebo-lymphology humanitarian trip to Matagalpa, Nicaragua}

\author{
Sergio Gianesini, ${ }^{1}$ Attilio Cavezzi, ${ }^{2}$ \\ Giovanni Mosti, ${ }^{3}$ Lorenzo Tessari, ${ }^{4}$ \\ Francesco Zini, 5 Simone Urso, ${ }^{6}$ \\ Fausto Campana, ${ }^{7}$ Mirko Tessari, ${ }^{1}$ \\ Patrizia Dalla Caneva, ${ }^{1}$ Freddy Espinoza, ${ }^{8}$ \\ Ruth Rocha, ${ }^{8}$ Diana Neuhardt, ${ }^{9}$ \\ Eric Mowatt-Larssen, ${ }^{10}$ Joe Zygmunt, ${ }^{11}$ \\ Susan Cortesi, ${ }^{12}$ Terri Morrison, ${ }^{12}$ \\ Nick Morrison ${ }^{12}$ \\ ${ }^{1}$ Vascular Disease Center, University of \\ Ferrara, Italy; ${ }^{2}$ Eurocenter Venalinfa, \\ San Benedetto del Tronto (AP), Italy; \\ ${ }^{3}$ Angiology Department, Clinica MD \\ Barbantini, Lucca, Italy; ${ }^{4}$ Glauco Bassi \\ Foundation, Trieste, Italy; ${ }^{5}$ Surgical \\ Department, Città di Parma Private \\ Hospital, Parma, Italy; ' ${ }^{\text {Phlebology }}$ \\ Department, Prof. Nobili Private \\ Hospital, Castiglione di Pepoli (BO), \\ Italy; ${ }^{7}$ Vascular Medicine, Bufalini \\ Hospital, Cesena, Italy; ${ }^{8}$ Fara Foundation, \\ Matagalpa, Nicaragua; \\ ${ }^{9}$ CompuDiagnostics, Phoenix, AZ, USA; \\ ${ }^{10}$ The Vein Specialists of Monterey, \\ Monterey, CA, USA; ${ }^{11}$ Veinz Phlebology \\ Consulting, Kure Beach, NC, USA; \\ ${ }^{12}$ Morrison Vein Institute, Tempe, AZ, \\ USA
}

\section{Abstract}

Amigos de Salud and Vene e Linfatici Foundation took part in a volunteer medical trip in Nicaragua. A detailed description of the provided healthcare is reported.

\section{Background}

Chronic venous disease (CVD) is an extremely widespread pathology, affecting up to $60 \%$ of women and $56 \%$ of men in the industrialized Caucasian race.

With the bias of extremely sparse literature about the topic, the population in limitedresource countries seem to be less prone to this disorder. ${ }^{1,2}$

Nevertheless, worrisome data in the few investigated resource-poor population have recently emerged regarding the most advanced stages of the disease, including venous ulcers. ${ }^{3}$

Under-estimated co-morbidities such as infections, diabetes, arterial disease and trauma have been identified as determinants of this burden. ${ }^{4}$

A dysfunctional lymphatic system and its pathophysiology are directly related to the venous pathophysiology. Even more than this, when lymphatic system is affected, skin infections may more easily occur, which eventually establishes a vicious circle in the venouslymph drainage impairment.

Phlebo-lymphatic disease (PLD) mismanagement, together with lack of appreciation of its co-factors, severely impacts patients in underdeveloped areas.

The real drama lies inside the relatively easy avoidance of this scenario by means of adequate disease management.

Indeed, severe PLD can lead to disabling conditions such as infections, impaired ambulation and even limb amputation. Moreover, considerable working days are lost, potentially severely impacting the social life.

Nevertheless, the modern scientific and therapeutic achievements of the industrialized countries have not yet reached the poor countries, leaving these populations in a vicious circle of severe impairment of quality of life and indeed economic distress.

\section{Amigos de salud activities}

Amigos de Salud is a non-profit organization, which for the last 25 years, by means of medical volunteer trips, has been providing diagnostic and therapeutic interventions for local people in Central and South American countries (Costa Rica, Ecuador, Nicaragua, Peru; http://www.amigosdesalud.org/).

The international medical team pays its own travel, covering all their own expenses, assembling all the needed equipment and providing industry contacts that can eventually support the poorest population healthcare, as well as scholarships and food programs.

Together with the medical team, invaluable help is provided by nurses, assistants, interpreters and family members of the healthcare team. All together this extra group becomes fundamental to the realization of the whole humanitarian act.

This year Amigos de Salud supported medical activities in Matagalpa, Nicaragua, in collaboration with the Italian Foundation Vene $e$ Linfatici, gathering some medical and nonmedical personnel dedicated to the care of venous and lymphatic diseases (Figure 1).

Thanks also to the conjunction with a local Foundation, an American/Italian team of eleven expert phlebologists was in charge of the treatment of $846 \mathrm{CVD}$ patients for a total of 942 lower limbs.
Correspondence: Sergio Gianesini, Vascular Disease Center, University of Ferrara, via Aldo Moro 8, 44128 Cona (FE), Italy.

E-mail: sergiogianesini@hotmail.com

Key words: volunteer, medical trip.

Conflict of interest: none

Acknowledgements: the volunteer members of Amigos de Salud and Vene e Linfatici Foundation express their deep gratitude for the passionate work that was done and that is still going on by $\mathrm{Dr}$ Ruth Rocha and Dr Freddy Espinoza, together with all the members of the local Fara Foundation (http://www.farafoundation.org), and it's directors, Marcela Cisne, Manny and Maria Farahani. Moreover, extreme gratitude is expressed also in favour of the Industries that supported the volunteer medical trip (in alphabetic order): Angiodynamics, Cook Medical, Eufoton, Farmax, Flebysan, Gloriamed, HK Surgical, Juzo, Kreussler, Lohmann-Rauscher, Medi, MyCli, Sigvaris, STD Pharmaceuticals, Techlamed, 3M, Trafita-Terason.

Received for publication: 30 November 2014. Accepted for publication: 29 January 2015.

This work is licensed under a Creative Commons Attribution 3.0 License (by-nc 3.0).

(C) Copyright S. Gianesini et al., 2015

Licensee PAGEPress, Italy

Veins and Lymphatics 2015; 4:4851

doi:10.4081/vl.2015.4851

\section{Amigos de salud and Vene e Linfatici Foundation 2014 medical support in Nicaragua}

\section{Population recruitment \\ and demographics}

In the months prior to the arrival of the volunteer medical group, two physicians of the local Nicaraguan Foundation screened 1000 patients coming from different regions of the country, exhibiting signs of CVD and complaining of the related symptoms.

Within this population, 117 patients presented with an active ulcer, of which 10 were bilateral.

The two local assessors excluded 50 patients because of major co-morbidities that were considered to significantly increase the risk of post-procedural complications.

On the patients' procedural day 104 patients missed the appointment because of unavailable economic funding to cover the trip to the hospital.

Of the residual 846 patients examined and treated by the Amigos de Salud team, 74 were males (8.74\%) and 772 were females (91.25\%) (Figure 2). The patients' age is reported in Table 1. 
Of the 846 treated patients, $688(81.32 \%)$ were never treated before, while 158 (18.67\%) had recurrent or residual disease.

\section{Management of the diagnostic and therapeutic process}

Over the course of the 6 working days the American-Italian team was able to provide all the following diagnostic and therapeutic procedures to the previously screened patients: i) specialist clinical and colour-duplex ultrasound investigation; ii) ultrasound-guided foam sclerotherapy; iii) preliminary saline flush followed by ultrasound-guided foam sclerotherapy; iv) endovenous laser ablation; v) laser assisted foam sclerotherapy; vi) catheter directed foam sclerotherapy; vii) ulcer debridement and advanced medication; viii) compression therapy; ix) hook phlebectomy.

In three cases a sapheno-femoral junction high ligation was performed without major or minor complications at one-month follow-up.

All the screened patients underwent a clinical and CDU assessment by the American/Italian team member. Patients then underwent the therapeutic procedure directly with the examiner physician, or were sent to colleagues who were ready to offer another kind of technical therapeutic option, whenever more appropriate for that specific case.

Once treated, in case of an ulcer presence, the patient was sent to the physician in charge of treating the ulcer itself.

The age of this ulcer subgroup population ranged from 30 to 84 years.

The ulcer etiology was: superficial CVD (36.9\%), deep CVD (25.8 \%), deep and superficial CVD (33\%), not determined (4.3\%).

The average ulcer area was $60 \mathrm{~cm}^{2}$ (ranging from 1 to $460 \mathrm{~cm}^{2}$ ), with an onset going back from 3 to 420 months. An active inflammatory ulcer was reported in $69.9 \%$ of cases, while $28 \%$ already presented granulation and $2.1 \%$ re-epithelization.

All the patients were treated by surgical debridement, advanced dressings and inelastic bandages.

A second wound healing session was possible and performed just in $8.6 \%$ of cases. All of them demonstrated improvement from the inflammatory to the granulation state.

In Table 2 numbers of performed procedures are reported.

Quite impressively, neither minor nor major peri-procedural complications were spontaneously reported by the patients, whereas two minor post-phlebectomy hematomas and a postsclerotherapy superficial thrombophlebitis were identified by the treating physicians.

\section{Post-procedural management}

A post-procedural elastic stocking of proper size, length (below or above-knee) and com-

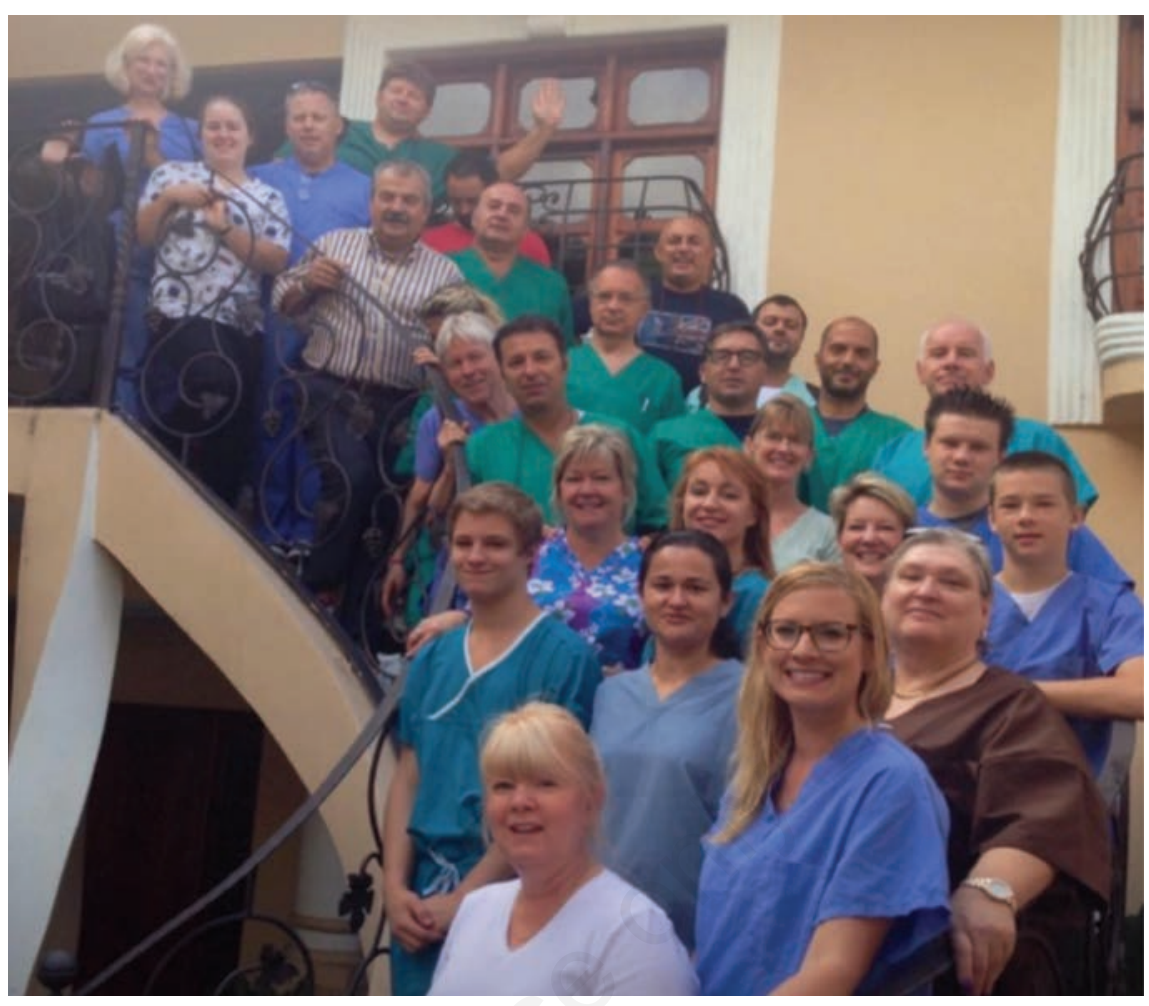

Figure 1. Amigos de Salud and Vene e Linfatici Foundation group.

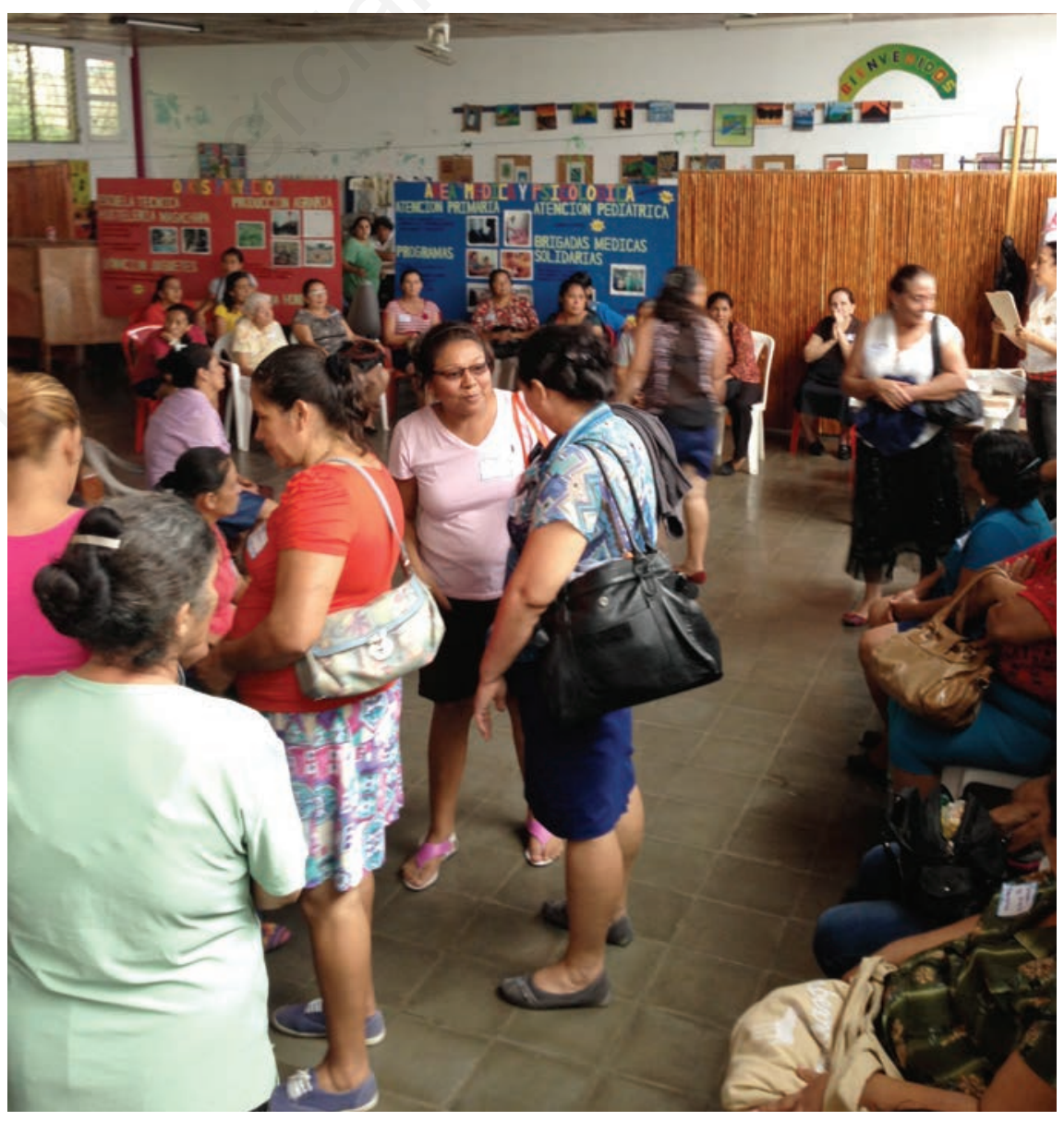

Figure 2. First patients joining the Fara Foundation clinic in early morning. 
pression class (15-20, 20-30, 30-40 mmHg) was donated and applied to all the patients (Figure 3).

After the treatments, the two Nicaragua physicians were available to follow-up and assist all the patients within the local Foundation clinic whenever needed, even after the American-Italian team's departure.

At one-month follow-up the two physicians were visited by 72 (7.6\%) patients.

The reasons for the patients returning to the clinic following the original treatment are reported in Table 3.

Further to the patients listed above, so far (November 2014) the doctors from the local Clinic are still weekly medicating and bandaging 75 out of the initial 117 active ulcers.

\section{Considerations on the Nicaragua 2014 experience}

Despite the short (6 days) commitment to the volunteer medical trip, the medical team was able to provide phlebological health-care to a large number of patients.

The $18.67 \%$ patients exhibiting residual/ recurrent disease illustrates the need to estab- lish local follow-up expertise, enabling the local physicians to treat signs and symptoms of recurrences at the very early stages. In this way it would be possible to complete the first therapeutic act, guaranteeing the longest disease-free time possible.
The $12.34 \%$ (104 out of 950 ) patients who had to drop out because of unavailable economic funding to cover the trip to the clinic is illustrative of the poverty afflicting these regions. These data highlight the need to provide further support to increase the potentially

Table 1. Treated population age.

\begin{tabular}{lcccccc} 
Age (years) & $14-25$ & $26-35$ & $36-49$ & $50-60$ & $61-74$ & $>75$ \\
& $0.7 \%$ & $8.3 \%$ & $32.2 \%$ & $30.9 \%$ & $22 \%$ & $5.9 \%$ \\
\hline
\end{tabular}

Table 2. Performed therapeutic procedures.

\begin{tabular}{lcc} 
Procedures & Number of treated cases & $\%$ \\
Ultrasound-guided foam sclerotherapy & 605 & 63.8 \\
Preliminary saline flush followed by ultrasound guided foam sclerotherapy & 45 & 4.7 \\
\hline Endovenous laser ablation & 96 & 10.1 \\
Laser assisted foam sclerotherapy & 39 & 4.11 \\
\hline Catheter directed foam sclerotherapy & 15 & 1.6 \\
Ulcer debridement, advanced medication & 117 & 12.3 \\
\hline Hook phlebectomy & 15 & 1.6 \\
High-ligation & 3 & 0.3 \\
\hline Compression (bandaging) & 130 & 13.7 \\
\hline
\end{tabular}

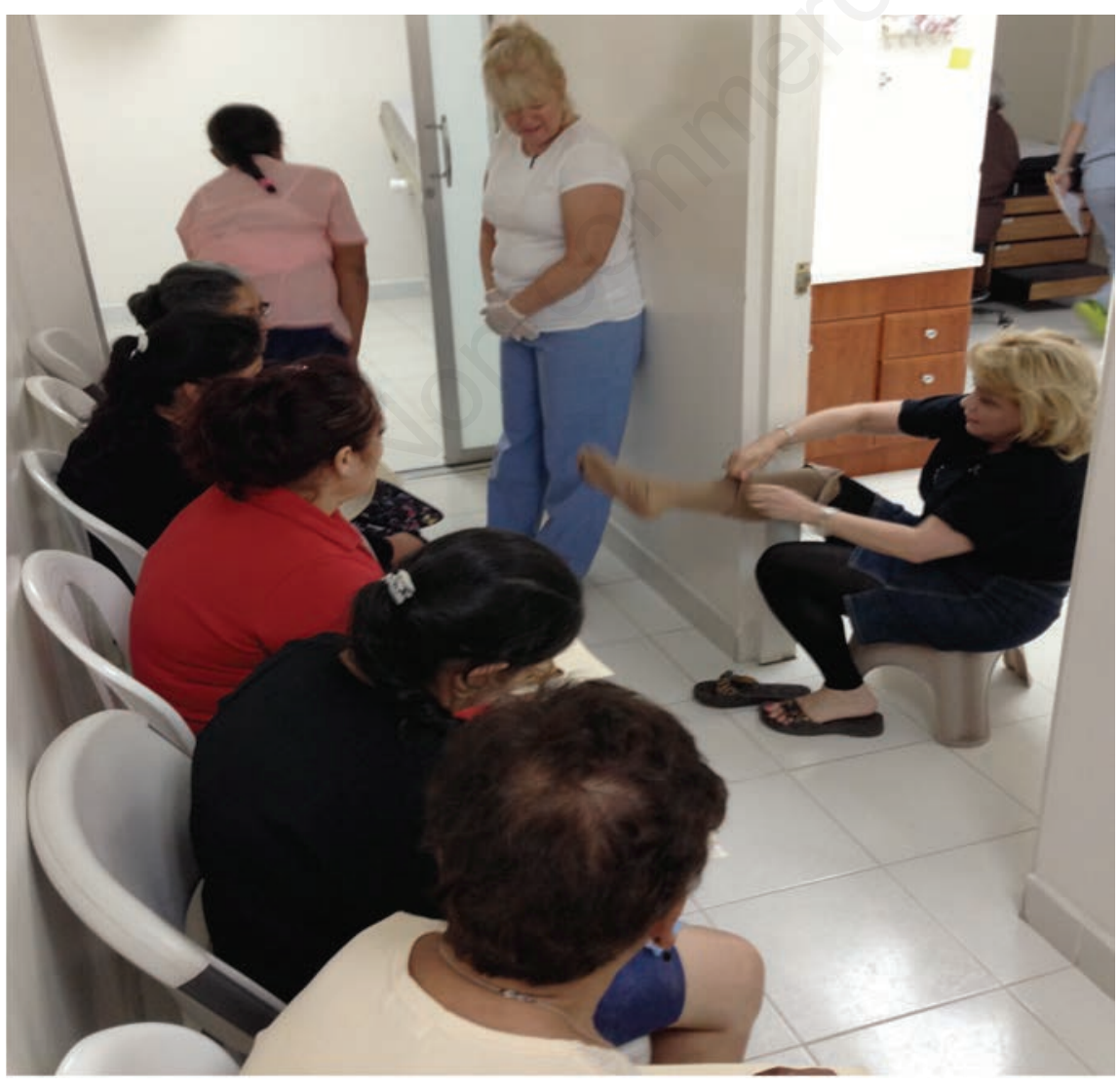

Figure 3. Amigos de Salud members teaching elastic stockings compression to the patients.
Table 3. Issues leading the patients to come back for a visit at one-month follow-up.

\begin{tabular}{lcc} 
Patient issue & Male & Female \\
Check-up & & 10 \\
Pain & 2 & 14 \\
\hline Superficial thrombophlebitis & 4 & 40 \\
Post procedural skin lesion & - & 2 \\
\hline
\end{tabular}

treatable population, which may benefit from such volunteer medical trips.

The presence of a well-trained and experienced medical and support team, together with an adequate fundraising campaign and the availability of very generous industry support allowed the creation of a temporary phlebological unit. A high level diagnostic and therapeutic approach was put in place in the local clinic, reaching standards typical of western country health providers.

\section{General considerations on volunteer medical trips}

In poorly resourced countries a major possible cause of drop out from the check up and treatment is the lack of nearby health struc- 
tures and obviously the lack of sufficient funds for the necessary travel to the clinic.

The local physicians who are involved in the patients' management all year long are particularly devoted to their humanitarian mission in favour of this poor population. Nevertheless, the overwhelming amount of work and the need to be a multi-specialty physician can result in complex management of all patients' needs especially their PLD.

Reports on social health data in underdeveloped countries are lacking, leading to an unclear scenario of the on-going critical situations and actual needs. ${ }^{5}$ This kind of data collection could also shed some light on those cofactors in PLD development that are not commonly known in industrialized countries.

\section{Conclusions}

The 2014 medical volunteer trip of the Amigos de Salud and Vene e Linfatici Foundation team to Matagalpa resulted in an effective and safe diagnostic and therapeutic approach to nearly 850 patients affected by various, mostly severe, stages of CVD.

Notwithstanding the objective limitations encountered during this experience, the medical and non-medical personnel was able to deliver proper care to 846 patients affected by varices, ulcers, lymphedema, post-thrombotic syndrome, venous malformations.

Further developments of similar humanitarian phlebology trips, such as adequate provisional of economical support for educational and diagnostic/therapeutic needs, are to be expected.

\section{References}

1. Beebe-Dimmer JL. The epidemiology of chronic venous insufficiency and varicose veins. Ann Epidemiol 2005;15:175-84.

2. Milic DJ. Prevalence and socioeconomic data in chronic venous disease: how useful are they in planning appropriate management?. Medicographia 2011;33:253-58.

3. Shukla VK, Mumtaz A, Gupta SK. Wound healing research: a perspective from India. Int J Lower Extremity Wounds 2005;4:7-8.

4. Nag F, De A, Hazra A, et al. Chronic venous ulceration of leg associated with peripheral arterial disease: an underappreciated entity in developing country. Int Wound $\mathbf{J}$ 2014;11:546-9.

5. http://chileangringo.blogspot.it/2009/05/ latin-america-gdp-pp.html 